\title{
INTERPRETATION OF PARAMAGNETIC MÖSSBAUER SPECTRA OF BIOLOGICAL MOLECULES IN TERMS OF THEIR ELECTRONIC STRUCTURE
}

\author{
G. LANG \\ Nuclear Physics Division, Atomic Energy Research Establishment, Harwell, England
}

\begin{abstract}
Résumé. - L'observation d'un certain nombre de composés de haem protéine à spin faible ou élevé dont la concentration en macromolécules est habituelle permet de déterminer leur structure électronique d'après l'étude qualitative des caractéristiques magnétiques du spectre Mössbauer. Dans de nombreux cas, un bon accord quantitatif est obtenu avec ce que prévoient des modèles électroniques simples. Les ambiguïtés d'interprétation intervenant dans les spectres électrostatiques sont nettement réduites par la complexité des caractéristiques magnétiques. A partir des informations obtenues pour des systèmes simples, la spectroscopie Mössbauer a fourni des informations précieuses sur des composés moins bien connus tels que les haems à spin divers, les peroxydases complexes, les ferredoxines et l'azote fixant les protéines bactériologiques.
\end{abstract}

Abstract. - Observation of a number of high and low spin haem protein compounds indicates that with the dilution common to macromolecule samples the electronic state usually can be determined by the qualitative behaviour of the magnetic features of the Mössbauer spectra. In many cases fair quantitative agreement is achieved with predictions based on simple electronic models, and the ambiguity of interpretation permitted in electrostatic spectra is much reduced by the complex magnetic features. With the background of information obtained in simple systems, Mössbauer spectroscopy has provided useful information in less understood materials, such as mixed spin haems, peroxidase complexes, ferredoxins, and nitrogen fixing bacterial proteins.

I. Introduction. - A typical biological macromolecule may have a molecular weight of $10^{4}$ to $10^{5}$, consisting for the most part of diamagnetic protein. It usually contains one or more regions which may be regarded as the centres of its biological activity. Often the centre is paramagnetic, containing a free radical or a transition metal ion. Various methods of magnetic measurement have been brought to bear on biological molecules, including susceptibility measurements, NMR, and ESR. More recently, Mössbauer spectroscopy has been used in the study of some of these materials. Almost all of the work has been concerned with the $14 \mathrm{keV}$ transition between the ground and first excited states of ${ }^{57} \mathrm{Fe}$, and we restrict ourselves to this system.

The Mössbauer spectrum is affected by both electrostatic and magnetic interactions. The electrostatic monopole interaction arises because the Mössbauer nucleus changes its charge radius in going from its ground state to its isomeric state. The resulting shift of the Mössbauer absorption energy (isomer shift) provides a measure of the local electronic charge density which is sometimes indicative of the valence state of the iron. The quadrupole moment of the excited nuclear state interacts with the electrostatic field gradient. Thus the spectrum is sensitive to asymmetry in the local electric charge distribution, being split into two absorption lines when such asymmetry is present. Magnetic fields, both internal and applied, can affect the spectrum through interaction with the magnetic moments of both the ground and excited nuclear states. These can give rise to complex spectra which often contain many lines.

In a typical paramagnetic salt only the electrostatic effects are normally observed in a Mössbauer spectrum, because rapid electron spin flip suppresses magnetic hyperfine interaction. As the result of considerable experimental effort the electrostatic spectra can in many cases be used to determine the valence state of the iron in the salt and the symmetry of its environment. The appearance of the spectrum is simple, being characterized by the two numerical values of isomer shift and quadrupole splitting, but its origins can be complicated, involving bonding as well as valence electrons and the charges of neighbouring ions. It is safe to say that their remains room for argument about the quantitative explanation of the electrostatic spectrum even in the simplest ionic salt. The situation is worse in the case of biological molecules, where there may be low symmetry, strong covalence, and less precise crystallographic information. It is fortunate that much additional information is contained in the magnetic hyperfine interactions and that the biomolecules, by virtue of the spin dilution provided by the large amount of protein, are especially suited for their observation. The magnetic features of the spectra reflect interaction with unpaired electron spin (which cannot always be treated as an effective field), and usually are of relatively complicated appearance. Their physical basis is simpler than is the electrostatic case because it is concerned only with the relatively few but chemically important unpaired electrons of the valence levels. Thus, in the cases where it is applicable, Mössbauer spectroscopy is a useful addition to the array of techniques for physical measurement of biological materials, and this is especially true when magnetic hyperfine interactions can be observed.

The first question usually asked about iron in a biological system is, «It is ferrous or ferric (or something else) ? », and "Is it high or low spin ?». From the magnetic features of the Mössbauer spectrum it is usually possible to make the related, and probably more precise, classification into zero, integral, and balf integral electron spin on the iron, and to distinguish $S=\frac{1}{2}$ from $S>\frac{1}{2}$. The zero spin materials exhibit no internal magnetic field. In the low symmetry environ- 
ment typical of biological molecules (and in fact virtually all molecules in general) integral spin systems have their spin degeneracy removed by electrostatic interaction, and internal magnetic hyperfine effects appear only in the presence of applied field which is strong enough to mix the electronic levels and induce a detectable magnetic moment. The molecules with half integral spin cannot have their spin degeneracy removed by internal electrostatic effects, and their magnetic nature manifests itself even in the absence of applied field, provided that the spin relaxation rate is sufficiently slow. This condition appears always to be satisfied for biomolecules at $4.2^{\circ} \mathrm{K}$, and many zero field magnetic spectra have been seen. Application of a field as small as a few gauss 'can cause marked alteration of the spectrum by decoupling electron spin from the nuclear spin. Once the applied field is large compared with the nuclear field at the electron (which is about 20 gauss in the typical iron case), increasing it has little effect upon the internal magnetic interactions until $\beta \mathrm{H}$ is comparable to the zero field splittings of the electronic states. These are typically several $\mathrm{cm}^{-1}$ when $S>\frac{1}{2}$, so that the magnetic mixing of electronic levels is observed. $S=\frac{1}{2}$ systems on the other hand have splittings of several hundred $\mathrm{cm}^{-1}$ and are readily distinguished. In the sections which follow, we will develop the theory of the magnetic hyperfine interaction and present a few examples in which the spectra calculated from simple models are seen to agree with the observations and with other kinds of magnetic measurements. Finally, some cases will be presented where Mössbauer spectroscopy appears to have contributed some new biologically relevant information.

II. Theory of the magnetic hyperfine interaction. A general treatment of magnetic hyperfine interaction in Mössbauer spectra, including relaxation effects, is given by Wickman [1]. We develop here only the part necessary for our later discussion. The Fe nucleus has a magnetic moment in both the ground and excited states. In the presence of unpaired electron spin in the slow relaxation limit the magnetic hyperfine interaction may be written as

$$
\begin{aligned}
\mathcal{H}_{\mathbf{M}}=P \sum_{K}\left\{l_{K} \cdot \mathbf{I}+3\left(\hat{\mathbf{r}}_{K} \cdot \mathbf{s}_{K}\right)\left(\hat{\mathbf{r}}_{K} \cdot \mathbf{I}\right)-\right. \\
\left.\quad-\left(\mathbf{s}_{K} \cdot \mathbf{I}\right)-K\left(\mathbf{s}_{K} \cdot \mathbf{I}\right)\right\}-g_{\mathbf{N}} \beta_{\mathbf{N}} \mathbf{I} \cdot \mathbf{H} \\
\left.P=2 g_{\mathrm{N}} \beta \beta_{\mathbf{N}}<\mathbf{r}^{-3}\right\rangle_{\text {effm }} .
\end{aligned}
$$

Here $\beta$ is theelectron magneton, $l$ is the orbital angular momentum of the $k$ th electron, $s_{k}$ is its spin, and the summation is taken over all unpaired electrons. The first term represents the interaction of the nucleus with electrons regarded as current loops, and the next two the come from ordinary magnetic dipole-dipole interactions. The term involving $K$ results from the Fermi contact interaction, and takes account of two effects : (1) the core s electrons are polarized through exchange effects with the unpaired $3 \mathrm{~d}$ electrons: (2) in suitable symmetries the $d$ electrons have a small amount of $4 \mathrm{~s}$ character. The last term in the Hamiltonian allows for the direct effect of any externally applied fied, $H$.

In the high spin ferric complex, which is in an orbital S state, only the contact term is important.
In the low spin ferric and high spin ferrous cases all three magnetic terms can be of comparable magnitude. The dipole term should not be neglected even in cubic systems, for it is zero from symmetry only in the absence of spin-orbit coupling. The quantity $\left\langle\mathbf{r}^{-3}\right\rangle_{\text {effm }}$ is an effective value which should be proportional to a shielding parameter analogous to the Sternheimer factor, and also to the covalency factor $\alpha^{2}$ of the unpaired electron. For a variety of high and low spin ferric haem compounds the present author has found $P=-4.2 \mathrm{~mm} \cdot \mathrm{s}^{-1}$ (for ${ }^{57 \mathrm{~m}} \mathrm{Fe}$ ), $K=0.35$ give reasonable fits to the data. In the Mössbauer energy units of ${ }^{57} \mathrm{Fe}, 1 \mathrm{~mm} . \mathrm{s}^{-1}$ corresponds to

$$
3.87 \times 10^{-4} \mathrm{~cm}^{-1} \text {. }
$$

The strength of the magnetic hyperfine interaction thus corresponds to a nuclear field of order ten gauss acting on the electrons, or an effective electron field of a hundred or a few hundred kilogauss acting on the nucleus.

The Hamiltonian (1) contains both electronic and nuclear operators, and operates on basis states which have both electronic and nuclear components. Because the hyperfine interaction is small compared with the typical zero field electronic energy level spacing, the electronic and nuclear parts of the problem usually can be solved separately when electron spin degeneracy is absent. In the more interesting case of ferric complexes, which must have half integral spin, the Kramers degeneracy prevents separation. Solution of (1) then corresponds to solving a spin coupling problem, analogous to $\mathrm{L}$. $\mathrm{S}$ coupling of atomic spectroscopy. An external magnetic field can be used to lift the Kramers degeneracy and again make the problem separable. The applied field need only be large compared with the field of roughly ten gauss which the nucleus exerts upon the electron. It is often convenient to express (1) in the formalism of the spin Hamiltonian as

$\mathscr{H}_{\mathrm{M}}=A_{x} I_{x} S_{x}+A_{y} I_{y} S_{y}+A_{z} I_{z} S_{z}-g_{\mathrm{N}} \beta_{\mathrm{N}} \mathbf{I} \cdot \mathbf{H}_{\mathrm{a}}$

where the operators $S_{x}$, etc. operate on effective spin. In the separable case they would be replaced by the corresponding spin expectation values.

Calculation of the Mössbauer absorption spectrum consists of then of the following several steps. 1) Solve the hyperfine Hamiltonian (including the electrostatic parts, which we have not discussed explicitly) for the system of nucleus plus electrons, find the sets of energy levels corresponding to both the excited and ground state nucleus. 2) Calculate the probabilities of all induced radiative transitions between the sets. The transition operator has in the present case a magnetic dipole nuclear component; its electronic part is a delta function, i. e. the electronic state does not change in the transition. 3) Fold into the resulting line absorption spectrum a Lorentzian characteristic of the energy widths of emitting and absorbing nuclei. For ${ }^{57} \mathrm{Fe}$ this has full width at half maximum of $0.2 \mathrm{~mm} . \mathrm{s}^{-1}$. Even at liquid He temperature, $k T$ is large compared with the hyperfine energies, but the thermal populations within the ground state set may have to be taken into account if electronic zero field or Zeeman splittings are of order $k T$. When external fields are applied to polycrystalline 
or solution specimens and additional average over field direction is necessary.

III. The high spin ferric complex. - The high spin ferric complex has five d electrons of parallel spin comprising a spherically symmetric subshell. The sixfold spin degeneracy is usually partially lifted by high order spin orbit coupling effects. These appear particularly effective in biological molecules, where the high asymmetry gives rise to rather large zero field splittings. As an example we consider the case of myoglobin fluoride [2], whose low temperature spectra, figure 1 , indicate the presence of internal fields of

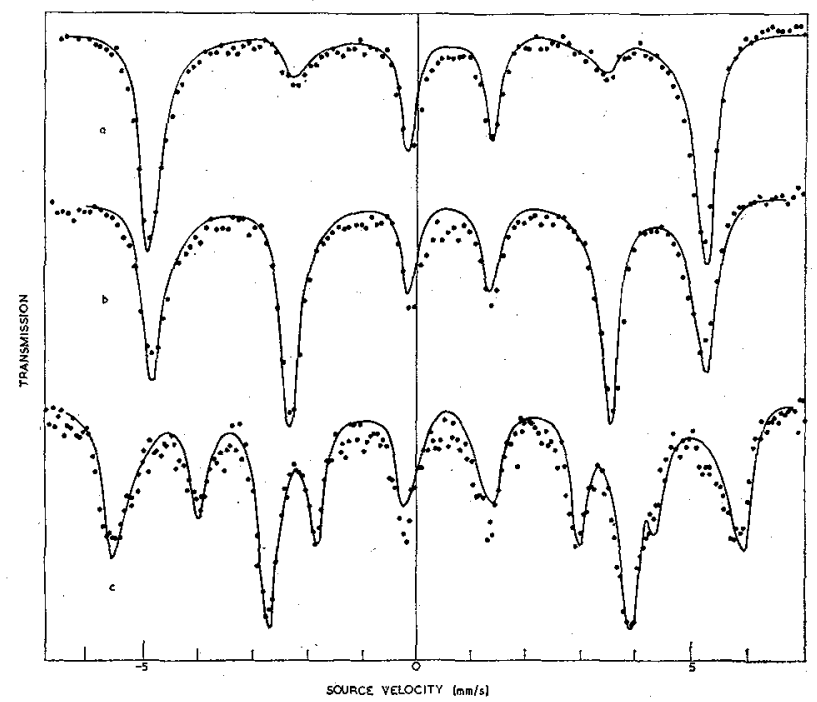

FrG. 1. - Mössbauer spectra of meso myglobin fluoride at $4.2 \% \mathrm{~K}$. Applied fields are (a) 0.1 kilogauss parallel to the gamma beam, (b) 0.5 kilogauss perpendicular to the gamma beam, (c) 10.2 kilogauss perpendicular. The solid curves are computed using an axial field model with $2 D=12.6 \mathrm{~cm}^{-1}, H_{s}=525 \mathrm{kilo}-$ gauss, $Q V_{z z} / 4=0.4 \mathrm{~mm} . \mathrm{s}^{-1}$, isomer shift relative to iron metal of $0.4 \mathrm{~mm} . \mathrm{s}^{-1}$, and a linewidth of $0.3 \mathrm{~mm} . \mathrm{s}^{-1}$ full width at half maximum.

roughly 300 kilogauss. These spectra are consistent with the spin Hamiltonian

$$
\begin{aligned}
\mathscr{H}=D[ & \left.S_{z}^{2}-S(S+1)\right]+2 \beta \mathbf{S} . \mathbf{H}+A \mathbf{I} . \mathbf{S}+ \\
& +\frac{1}{4} Q V_{z z}\left[I_{z}^{2}-I(I+1)\right]-g_{\mathrm{N}} \beta_{\mathrm{N}} \text { I.H. }
\end{aligned}
$$

The term in $D$ causes zero field splitting into three doublets as shown in figure 2 . At $4.2^{\circ} \mathrm{K}$ only the lowest doublet is occupied. Application of an external field splits the ground doublet into states whose spin expectation values depend on the field direction. As the figure indicates, however, the behaviour averaged over molecular orientation is very close to that produced by $H$ lying in the $x y$ plane, and we can explain the spectra qualitatively by considering only this case. For small $H$, both members of the doublet have $|\langle\mathbf{S}\rangle|=\frac{3}{2}$. The electronic and nuclear parts of the Hamiltonian may be separated and the interaction of the spin with the nucleus is equivalent to an effective field

$$
\mathbf{H}_{\text {eff }}=A<\mathbf{S}>/ g_{\mathrm{N}} \beta_{\mathrm{N}}=H_{\text {sat }}<\mathbf{S}>/(5 / 2) .
$$

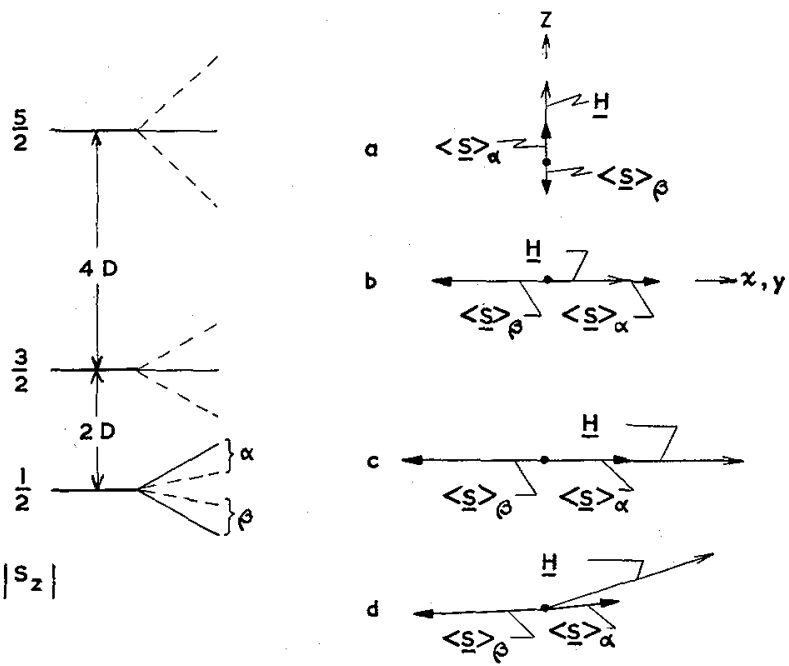

FIG. 2. - Energy level scheme of the high-spin ferric complex with axial crystal field (left). Dotted lines show splitting in axial magnetic field, while solid lines show the effects of transverse field. To the right are shown the spin-expectation values of the components of the ground doublet in applied field. The first case, field along ${ }^{3}$, has low statistical probability in a polycrystal, and the spectrum (e. g. Fig. $1 a, 1 b$ ) is determined by conditions near to those of the second case. In the third case, large $H$, the components of the doublet have different values of $\mid\langle S\rangle$, giving rise to separate six line spectra, as in figure 1c. The values of $|\langle S\rangle|$ are only slightly affected when the field is inclined to the $x y$ plane, as in the lowest example (Ref. 2).

Since the members of the lowest doublet have equal and opposite spin expectation values they give rise to similar Mössbauer spectra, and only a single magnetic pattern is seen in figures $1 a$ and $1 b$. The curves shown are computed averages over all orientations, taking $H_{\text {sat }}=525$ kilogauss. Larger fields can cause mixing of the electronic doublets, giving rise to different values of $\langle\mathbf{S}\rangle$, hence different $H_{\text {eff }}$, for the lowest two states. In this case the spectrum is seen to consist of two patterns corresponding to unequal fields. Their different intensities reflect the thermal populations of the electronic levels. The computed curve of figure $1 c$ is based on a $D$ value of $6.3 \mathrm{~cm}^{-1}$, in essential agreement with the value $6.5 \mathrm{~cm}^{-1}$ obtained by Kotani [3] from susceptibility measurements.

The fourth term of (4) is the quadrupole interaction, here assumed axial, which has been taken to be $Q V_{z z} / 4=0.4 \mathrm{~mm} \cdot \mathrm{s}^{-1}$ in order to reproduce the asymmetry of the spectra of figure 1 . The quadrupole splitting in the absence of magnetic interactions would provide a check of the value and the axial symmetry assumption. Unfortunately the magnetic effects persist to high $T$ in myoglobin fluoride, so that the quadrupole interaction cannot be seen alone. In the analogous case of acid metmyoglobin the $195^{\circ} \mathrm{K}$ spectrum is a quadrupole doublet, and is consistent with the axial model which fits the low $T$ data [2].

A number of high spin haem proteins have been measured (see page 48 of the monograph of Lang [4] for a tabulation), the fluorides having $H_{\text {sat }}$ near 520 kilogauss and $D$ about 7 kilogauss. The acid met complexes are also consistent, with $H_{\text {sat }}$ about 490 kilogauss, $D$ about $10 \mathrm{~cm}^{-1}$. Results have been generally consistent with other types of measurements, one noteworthy exception being acid met myoglobin whose 
$D$ is found to be $10 \mathrm{~cm}^{-1}$ by Mössbauer spectrometry [2] and by susceptometry [3], while the independent ESR results of Eisenberger [5] and Slade [6] both indicate $D=4.4 \mathrm{~cm}^{-1}$.

The zero field low $T$ spectra of all high spin haems and most high spin complexes in general are very much affected by covalent transfer of electron spin to ligands and the resulting hyperfine interaction with their nuclei. These coupled spin systems are very complicated and the spectra are not very promising sources of electronic information.

IV. The low spin ferric complex. - A typical low spin ferric haem protein exhibits a simple quadrupole spectrum at dry ice temperature and above. At $77^{\circ} \mathrm{K}$ this is usually made asymmetric by magnetic interaction with the electrons spins, which have intermediate relaxation time at this temperature. At $4.2{ }^{\circ} \mathrm{K}$ the spectrum is already at the slow relaxation rate limit and a complicated magnetic spectrum appears. Application of a small external field modifies it considerably by spin decoupling. It has been possible to account for the spectra in most cases with a rather simple model which we shall outline below.

In the low spin ferric system, five electrons occupy the six available states made up of orbitals $\mid x y>$, $|x z\rangle$, and $|y z\rangle$ combined with spin states $\alpha$ and $\beta$. In our simple model we consider other orbital levels to be infinitely far away in energy, and characterize the system by two splittings as shown in figure 3 . In the

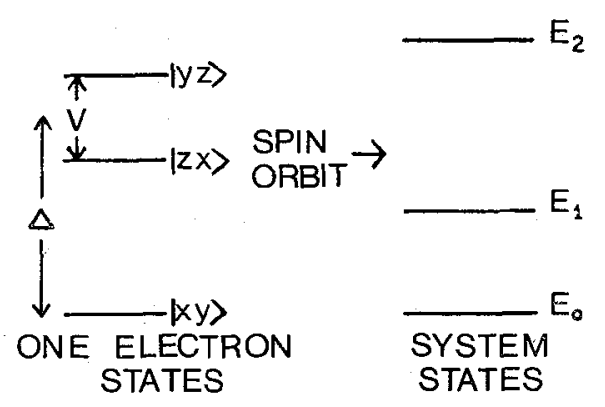

Fig. 3. - Low spin ferric energy level diagram. At the left are the one electron levels of $t_{2 g}$, which combine with spin functions to make six states. In the absence of LS coupling these would be occupied except for a single hole in $|y z\rangle$. In the actual situation the hole is distributed over all three orbitals, and each system wave function is a linear combination of three five-electron determinental wave functions.

absence of LS coupling we would have three doublets corresponding to the placing of the hole in each of the orbitals. In the presence of this interaction, however each eigenstate is a linear combination of these. The combining coefficients, and hence the properties of the eigenstates, are functions of $\mathrm{V} / \zeta$ and $\Delta / \zeta$, where the splittings are as shown in figure 3 and $\zeta$ is the spinorbit coupling constant. $\zeta$ is typically of order $300 \mathrm{~cm}^{-1}$, and the separation between the eigenstate doublets will be at least of this order no matter what values $V$ and $\Delta$ assume. Thus at the low temperature necessary for slow spin relaxation, and in available laboratory magnetic fields we need consider only the ground doublet. Solution of the spin-orbit problem and subsequent application of (1) makes possible the evaluation of $A$, the magnetic hyperfine coupling tensor of (3). Detailed formulae are given in reference 7 . Since the ESR $g$ values may also be expressed in terms of $V / \zeta$ and $\Delta / \zeta$, the Mössbauer spectrum can be used to predict the $g$ values and vice versa.

We show as an example the spectra [4] of cytochrome $c$ of $T$. Utilis in figures 4 and 5 , taken at $4.2^{\circ} \mathrm{K}$

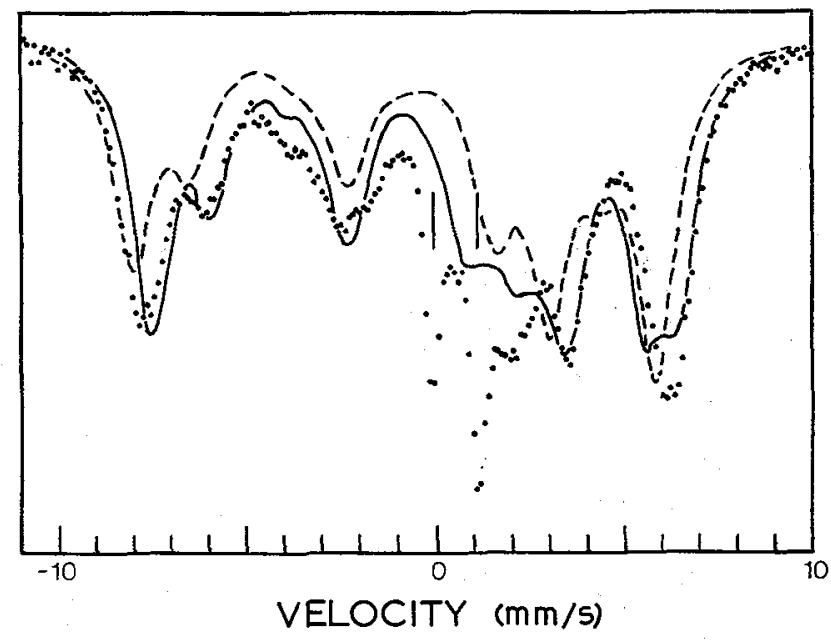

FIG. 4. - Mössbauer spectrum of ferricytochrome $\mathrm{c}$ of $\mathrm{T}$. utilis in absence of applied magnetic field at $4.2 \mathrm{oK}$. The solid curve is the calculated spectrum based on $g$ values $(1.24,2.25$, 3.07 ) which are in essential agreement with those of horse heart ferricytochrome $\mathrm{c}$ (Ref. 8). The dashed curve corresponds to $g=(1.0,2.4,3.0)$ and provides an indication of the relative sensitivity of Mössbauer spectroscopy. A line width of $1.2 \mathrm{~mm}$. $\mathrm{s}^{-1}$ has been assumed in the calculations to take rough account of relaxation effects, transferred hyperfine interaction with ligand nuclei, and distortion of the frozen protein. Vertical lines mark absorption of ferrous cytochrome (Ref. 4).

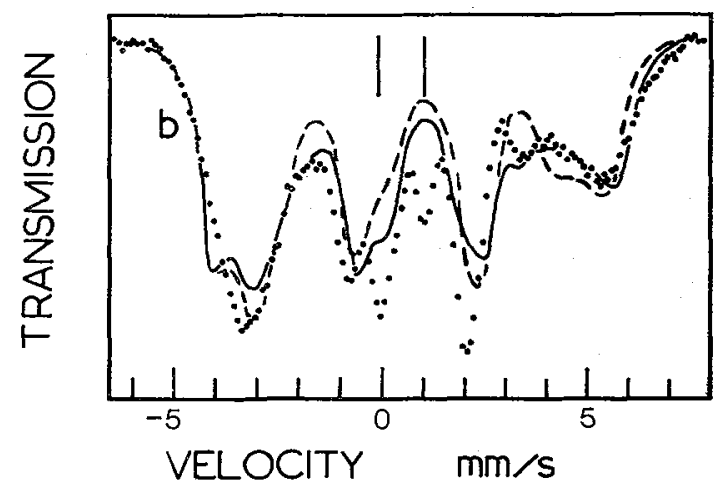

Fig. 5. - Mössbauer spectrum of ferricytochrome c of $T$. utilis in a transverse magnetic field of 500 gauss at $4.2 \mathrm{oK}$. The solid curve is the calculated spectrum based on $g=(1.24$, $2.25,3.07$ ), near that of horse heart ferricytochrome c (Ref. 8). The dashed curve corresponds to $g=(1.0,2.4,3.0)$, and provides an indication of the relative sensitivity of Mössbauer spectroscopy. A line width of $0.6 \mathrm{~mm} . \mathrm{s}^{-1}$ has been assumed in the calculations to take rough account of relaxation effects and distortion of the frozen protein. Vertical lines mark absorption of ferrous cytochrome (Ref. 4).

in zero and 500 gauss field respectively. The solid calculated curves are based on Salmeen's [8] measured $g$ values of horse heart cytochrome c. (Species dependence in the $g$ value is usually small.) The magnetic scaling parameters are given above; the quadrupole interaction with $3 \mathrm{~d}$ electrons was taken such that an electron in a pure $|x y\rangle$ orbital would give a splitting 
of $3 \mathrm{~mm} \cdot \mathrm{s}^{-1}$. In contrast to the high spin case, the simple model which couples only the iron nucleus to the unpaired electrons appears to give a fair approximation of the data. Ligand nuclei interactions may be responsible for the large apparent line width, but they are an order of magnitude smaller than in the high spin case. The crystal field splittings in this case are

$$
V / \zeta=1.47, \Delta / \zeta=2.56
$$

V. Some examples of new results. - The examples of the previous sections could be regarded as confirming information already available from other types of measurements. We now consider briefly some situations in which Mössbauer spectroscopy has provided information about biological materials which was not previously known, and which at least in some cases has direct biological significance.

An interesting situation arises when the $g$ values of a $S=\frac{1}{2}$ complex are very near to the free spin values. In the model above this could occur if the crystal field splittings were zero, $\mathbf{i}$. e. if the system had cubic symmetry. (Actually, the signs of the $g$ values would be opposite to the free spin value in this case, but $g$ signs ordinarily are not determined.) Alternatively, $g$ near the free spin value would occur if a single unpaired electron were located in an orbital level which was separated from its neighbours by energies large compared with $\zeta$. In this case it would be of interest to know if the orbital were of sigma type (as $\left|3 z^{2}-r^{2}\right\rangle$ ) or of pi type (as $|x y\rangle$ ). The low temperature magnetic Mössbauer spectrum could distinguish the three cases because it senses the tensor, whose qualitative features are different in each case. In the cubic case, all components of $A$ would be equal. In the isolated orbital case we would have $L=0$, and can find A by considering contact and dipole contributions only. As figure 6 shows, the dipolar and contact hyperfine interactions tend to cancel when the electron position vector is parallel to its spin, while they add in the broadside position. In the sigma orbital, two spin directions correspond predominantly to the broadside arrangement and one to the parallel arrangement, and thus the $\mathrm{A}$ tensor has two large and one small component. For the pi orbital the broadside arrangement holds only for spin perpendicular to the plane of the orbital, hence there is only one large component of $\mathrm{A}$. In the presence of a small spin decoupling field the distinction between the three cases is clear even when polycrystalline specimens are used. In the cubic case there is a single value of effective internal field, and a spectrum with well defined structure is expected. In the pi orbital case, the internal field is near its maximum value for most orientations (see the probability distribution plot in figure 6) and a spectrum has less well defined lines. For the sigma orbital, all internal field values up to the maximum are of nearly equal probability, and a smeared spectrum results. The spectrum of haemoglobin nitric oxide, figure 7, indicates that its unpaired electron is in a sigma orbital. The computed spectrum of the figure 7 corresponds to the electronic scheme shown in figure 8, where the pi and sigma bonding electrons are assumed to be shared equally between the iron and the nitrosyl nitrogen.

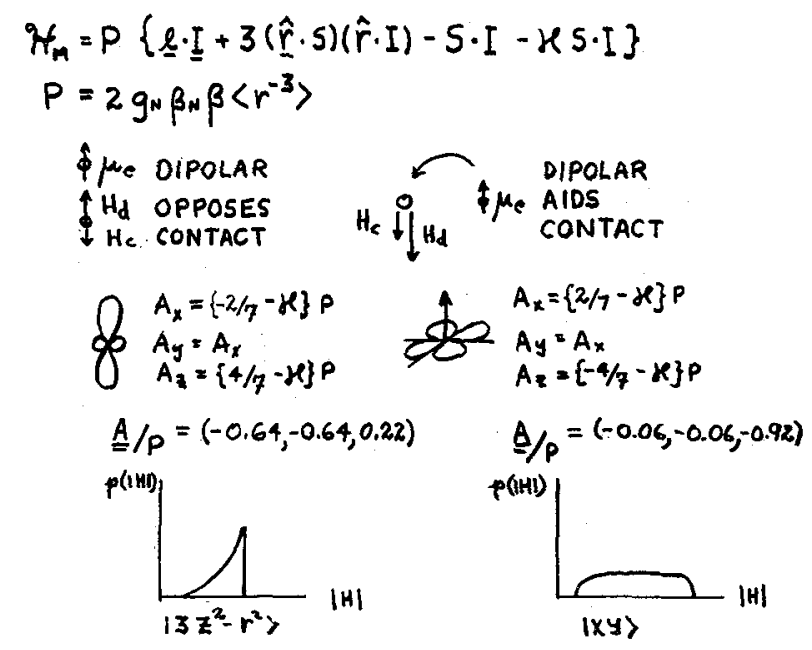

FIG. 6. - Diagram explaining qualitative features of Mössbauer spectra caused by unpaired electron spins in isolated orbitals. The sigma orbital is elongated (left side), and when the electron moment $\mu$, lies parallel to it the opposition of dipolar and contact interactions makes the net magnetic interaction with the nucleus small. When $\mu_{\mathrm{e}}$ is transverse to the length of the orbital the two terms add. Thus the $A$ tensor has one small and two large components. When an applied field forces $\mu_{\mathrm{e}}$ to assume all possible directions in a polycrystalline sample the statistical preference for transverse directions means that at most nuclei the effective field will be near its maximum value of $A_{i} g$, and a spectrum with well-defined peaks will result. For the pi orbital A will be large when $\mu_{\mathrm{e}}$ lies perpendicular to the plane of the orbital. Since $H_{\text {eff }}$ is then approximately proportional to $\cos \theta$, the result is an almost uniform distribution of $H_{\text {eff }}$ values between zero and the maximum.

The result will be a spectrum without well-defined lines.

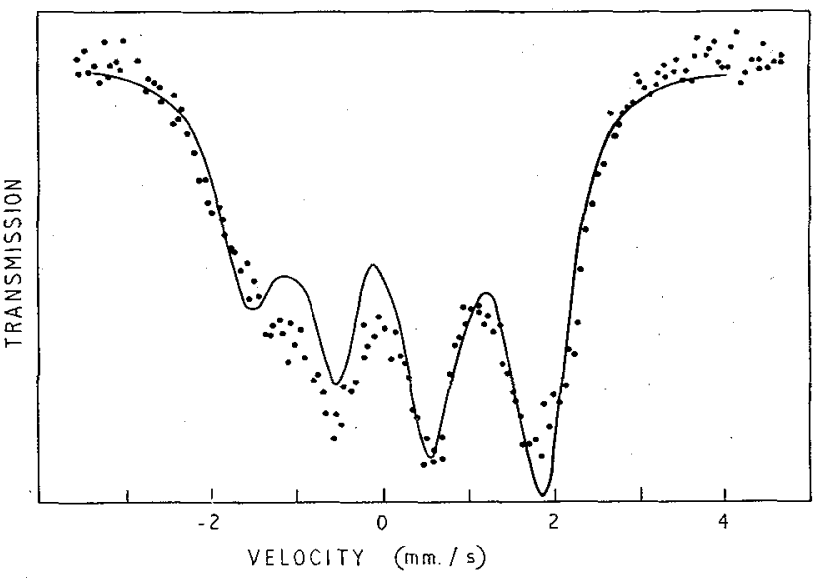

FIG. 7. - Mössbauer spectrum of haemoglobin nitric oxide at $4.2 \mathrm{~K}$ in a magnetic field of 500 gauss transverse to the gamma beam. Both pi bonds (see Fig. 8) and the sigma bond, which contains the unpaired electron, are assumed to have an iron component which is about half that of a typical low-spin haem protein. The figure is from Ref. 9.

In systems where little is known of the state of the iron, even rough qualitative features of the Mössbauer spectrum can be of considerable significance. Interesting examples are the peroxidases, which function to combine with and decompose hydrogen peroxide. Two examples of this class are horseradish peroxidase (HRP) and cytochrome $c$ peroxidase (CCP). Ferric HRP combines with hydrogen peroxide to form a 


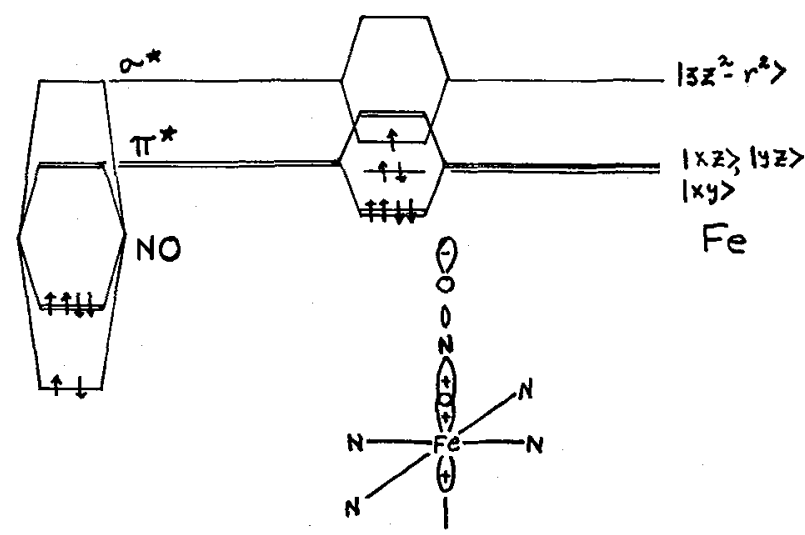

FIG. 8. - Energy level diagram which is consistent with Mössbauer spectrum of haemoglobin nitric oxide.

green complex I with two extra oxidizing equivalents. One of these is subsequently lost in a spontaneous conversion to a red complex II. Mössbauer measurements by Moss et al. [10] show isomer shift of $0.03 \mathrm{~mm}$. $\mathrm{s}^{-1}$ for II, and I is not significantly different. Thus the iron is similar to that in Fe IV inorganic complexes, and one of the oxidizing equivalents must be remote from the iron. ES, the closely similar peroxide complex of $\mathrm{CCP}$, has two equivalents above $\mathrm{Fe}^{3+}$, and a similar spectrum. Electron spin resonance measurements of Yonetani and Schleyer [11] and susceptibility measurements of Iizuka et al. [12] suggest the presence of both integral and half integral spin. Electronic magnetic effects are seen in Mössbauer spectra of Lang et al. [13] only in the precence of an applied field, indicating that the integral spin resides on the iron, but the half integral one does not. The work on this family of enzymes is still in progress, but we can see here some examples of Mössbauer spectroscopy being used on several levels of complexity. The experiment in which nothing happened to the iron in the course of a chemical change of the molecule is the simplest and most significant, implying an electron accepting site not directly on the iron or its near neighbours. The picture is supported and extended by the demonstration that a half integral spin (detected by ESR) is not near the iron. This spin is almost certainly located on the site in question. On a more quantitative and mathematical level, it is beginning to appear that the combination of electric and magnetic features sensed by the ${ }^{57} \mathrm{Fe}$ nucleus can be explained in terms of a reasonable molecular orbital model which exhibits the proper susceptibility as well. While this does not identify the group attached to the iron, it can provide a criterion for choosing among possibilities which other considerations might suggest.

As a final example we consider a recent Mössbauer study by Kelly and Lang [14] of bacterial nitrogenase. This consists of two proteins, both containing iron and one containing molybdenum as well. When mixed together and provided with a suitable supply of electrons (sodium dithionite) and energy (in the form of ATP) the system is capable of splitting the nitrogen molecule and forming $\mathrm{NH}_{3}$. Each protein has a distinctive Mössbauer spectrum, and mixtures yield a simple sum spectrum unless both materials necessary for fixation are present. In this condition new components appear in the Mössbauer spectrum, suggesting very strongly that the iron, or some of it, is at an active centre or centres. A second interesting feature is seen in figure 9, where the $4.2 \mathrm{~K}$ spectra of the FeMo

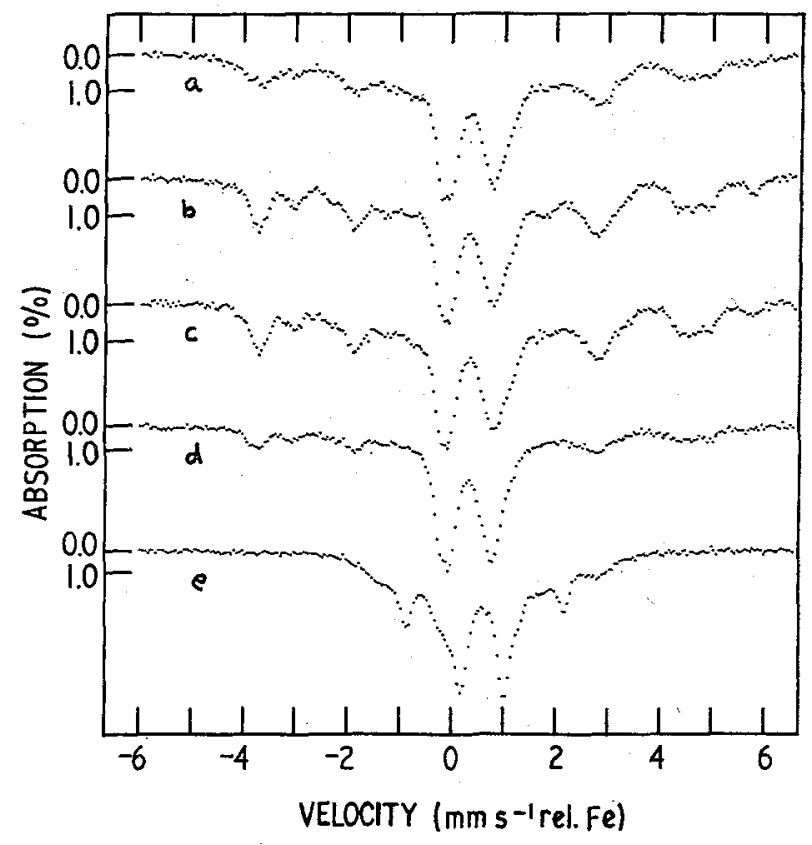

Fig. 9. - Spectra at $4,2 \mathrm{oK}$ of the FeMo protein of nitrogenase/ The first three are from an argon flushed sample containing $4 \mathrm{mg}$ of $\mathrm{Na}_{2} \mathrm{~S}_{2} \mathrm{O}_{4}$ with $(a)$ no magnetic field $(b)$ a field of about 100 gauss parallel to the gamma beam, and $(c)$ a field of 550 gauss transverse to the gamma beam/ Removal of dithionite by dialysis results in spectrum $(d)$, obtained in 550 gauss transverse field. The sample responsible for $(e)$ in 550 gausswas undialysed material incubated for one hour under argon at $20^{\circ} \mathrm{C}$, with $20 \mathrm{mg}$ of dithionite per ml. of solution.

protein indicate the presence of half integral spin. The similarity of $9 b$ and $9 c$ indicates that the spins which produce the internal magnetic field are insensitive to the direction of the applied field. This suggests that the operators $S_{x}$ and $S_{y}$ have zero matrix elements, a property which can occur in some of the doublets of a system with $S>\frac{1}{2}$. On the other hand the strength of the magnetic hyperfine interaction seems small for intermediate or high spin iron. The protein is normally prepared in weak dithionite solution, and curve $d$ shows that the magnetic part of the spectrum is decreased in intensity when the dithionite is removed. This appears odd, for we would normally expect such a move in the oxidizing direction to increase the amount of half integral spin (i. e. ferric) iron. A likely conclusion is that the iron atoms exist in pairs which are spin coupled in the antiferromagnetic sense. If one is ferrous, the unpaired spin is shared between the atoms, giving rise to the relatively weak magnetic hyperfine interaction. When both irons of a pair are ferric a nonmagnetic combined state could be formed. The fixing system must somehow collect the electrons from the reductant and another well known electron transfer agent, ferredoxin, is known to contain iron pairs which share electron spin. The nitrogenase is not a ferredoxin, however, in that the shared spin of the latter has clearly the value $\frac{1}{2}$. 
It is fortunate that iron, which is rather wide spread in living systems, is a very convenient Mössbauer nucleus. It is fortunate as well that the magnetic dilution of biological systems facilitates the study of its magnetic hyperfine interaction. We may reasonably. expect this new spectroscopy to contribute significantly to the understanding of biomolecules which fall within its scope.

\section{References}

[1] Wickmann (H. H.) and Werthem (G. K.), in Chemical applications of Mössbauer Spertroscopy, Academic Press, New York, 1968.

[2] Lang (G.), Asakura (T.) and Yonetani (T.), Biochim. Biophys. Acta, 1970. 207, to be published.

[3] Kotani (M.) and Morimoto (H.), in Magnetic Resonances in Biological Systems, 1967, 135. Oxford and New York, Pergamon Press.

[4] Lang (G.), Quart. Rev. Biophys., 1970, 3,1. J. Chem. Phys., 1967, 47, 3327.

[6] Slade (E. F.) and Ingram (D. J. E.), Proc. Roy. Soc. London, 1969, A 312, 85.

[7] Lang (G.) and Marshall (W.), Proc. Phys. Soc., 1966, 87, 3.

[8] Salmeen (I.) and Palmer (G.), J. Chem. Phys., 1968, 48, 2049 .

[9] Oosterhuis (W. T.) and Lang (G.), J. Chem. Phys., $1969,50,4381$.

[10] Moss (T. H.), Ehrenberg (A.) and Bearder (A. J.), Biochem., 1969, 8, 4159.

[11] Yonetani (T.) and Schleyer (H.), J. Biol. Chem., $1967,242,3919$.

[12] Izzuka (T.), Kotani (M.) and Yonetani (T.), Biochim. Biophys. Acta., 1968, 167, 257.

[13] Lang (G.), Asakura (T.) and Yonetani (T.), unpublished results presented at Fourth International Conference on Magnetic Resonance on Biological Systems, Oxford 1970.

[14] Kelly (M.) and LANG (G.), Biochim. Biophys. Acta, to be published. 